\title{
THE GEOMETRY OF WHIRLS AND WHIRL-MOTIONS IN SPACE ${ }^{1}$
}

\author{
J. M. FELD
}

1. Introduction. The geometry of whirls and whirl-motions in the plane was inaugurated by E. Kasner. ${ }^{2}$ An adaptation of Kasner's geometry to the sphere was made by K. Strubecker. ${ }^{3}$ It is the purpose of this paper to develop a strictly analogous geometry in euclidean three-space, $S_{3}$. To render such a development possible we shall introduce a new type of oriented plane element-namely, a geometric object formed by a plane, a point in the plane, and an ordered pair of orthogonal fundamental directions in the plane; the fundamental directions shall be given by a pair of unit vectors in the plane. There are $2 \infty^{6}$ such plane elements in $S_{3}$; there are $\infty^{5}$ of the Lie kind. Henceforth, plane element shall mean only the new kind of plane element. ${ }^{4}$

2. Turns, slides, and direct whirls. Let $e_{0}, e_{1}, e_{2}, e_{3}$ be the Hamiltonian quaternion units such that

$$
\mathfrak{e}_{0} \mathrm{e}_{i}=\mathrm{e}_{i} \mathrm{e}_{0}=\mathrm{e}_{i}, \quad \stackrel{2}{\mathrm{e}_{1}}=\stackrel{2}{\mathrm{e}_{2}}=\stackrel{2}{\mathrm{e}_{3}}=\mathrm{e}_{1} \mathrm{e}_{2} \mathrm{e}_{3}=-1 .
$$

Let any real point $P$ in $S_{3}$ whose orthogonal cartesian coordinates are $z_{1}, z_{2}, z_{3}$ be represented by the position vector $z=z_{1} e_{1}+z_{2} e_{2}+z_{3} e_{3}$. Let

${ }^{1}$ Presented to the Society February, 22, 1941.

${ }^{2}$ Edward Kasner, The group of turns and slides and the geometry of turbines, American Journal of Mathematics, vol. 33 (1911), pp. 193-202. Further development of the subject appears in a series of papers by Kasner and De Cicco; see, for example, their joint papers, Quadratic fields in the geometry of the whirl-motion group $G_{6}$, ibid., vol. 61 (1939), pp. 131-142; and The geometry of the whirl-motion group $G_{6}$ : elementary invariants, this Bulletin, vol. 44 (1938), pp. 399-403.

${ }^{3} \mathrm{~K}$. Strubecker, Zur Geometrie sphärischer Kurvenscharen, Jahresbericht der Deutschen Mathematiker-Vereinigung, vol. 44 (1934)', pp. 184-198.

${ }^{4}$ Inasmuch as our oriented plane element defines a position of a rigid body in space, it is essentially equivalent to Study's soma, Geometrie der Dynamen, Leipizg, 1903, and to De Saussure's feuillet, Exposé Résumé de la Géométrie des Feuillets, Geneva, 1910; see also R. Bricard, Nouvelles Annales de Mathématiques, (4), vol. 10 (1910). It was remarked by Kasner in his 1911 paper, loc. cit., that it was possible to obtain in any space of constant curvature a group analogous to his group of whirls in the plane and that, moreover, for ordinary space the feuillet, consisting of a point, line, and plane all incident with one another, would be an appropriate element. Another generalization of Kasner's turbine geometry, along lines different from those pursued in this paper, has been carried out by A. Narasinga Rao, Studies in turbine geometry I, Journal of the Indian Mathematical Society, vol. 3 (1938), pp. 96-108; II, Proceedings of the Indian Academy of Sciences, vol. 8A (1938), pp. 179-186. 
$O R$ be an axis through the origin parallel to a given unit vector $v$, and let

$$
\zeta=-\cos \theta / 2+v \sin \theta / 2, \quad \bar{\zeta}=-\cos \theta / 2-v \sin \theta / 2 .
$$

Then the rotation of $z$ around $O R$ through the angle $\theta$ is given by Hamilton's formula ${ }^{5}$

$$
z^{*}=\bar{\zeta} z \zeta
$$

We shall refer to $(2.1)$ as the rotation $\zeta$.

Let $\&$ be a plane element characterized by the point $z$ lying in the plane $P$ and the pair of fundamental orthogonal directions given by the unit vectors $v_{1}, v_{2}$, in this order. Let $\zeta$ be the (unit) quaternion by means of which the vectors $\mathfrak{e}_{1}$ and $\mathfrak{e}_{2}$ can be rotated around an axis passing through the origin into positions parallel respectively to the directions of $v_{1}$ and $v_{2}$. There evidently exists a $(1,1)$ correspondence between the plane elements $\&$ and the pairs of quantities $z$, $\zeta$; we shall, accordingly, call $z, \zeta$ the coordinates of $\&$, and shall use the symbol $(z, \zeta)$ to designate the plane element whose coordinates are $z, \zeta$.

Definitions. A slide is a plane element transformation, $(z, \zeta)$ $\rightarrow\left(z^{*}, \zeta^{*}\right)$, which leaves the planes of the plane elements unaltered, but subjects their points to the same translation relative to their fundamental directions.

A turn is a plane element transformation which leaves the points of the plane elements unaltered, but rotates their planes through the same angle around an axis inclined in the same manner relative to their fundamental directions.

$A$ direct whirl ${ }^{6}$ is a plane element transformation resulting from the

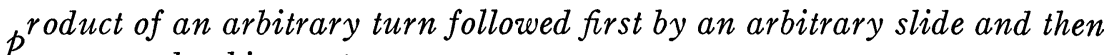
$b$ y a second arbitrary turn.

These definitions are the space analogues of those given by Kasner or the plane.

Let $a=a_{1} e_{1}+a_{2} e_{2},\left(a_{i}\right.$ real $)$; then the slide $(z, \zeta) \rightarrow\left(z^{*}, \zeta^{*}\right)$, which shall be represented by $S_{a}$, is given by the equations:

$$
z^{*}=z+\bar{\zeta} a \zeta, \quad \zeta^{*}=\zeta .
$$

${ }^{5}$ It will suffice for our purpose to restrict ourselves throughout this paper to unit quaternions - that is, those having a norm equal to unity.

${ }^{6}$ The term direct whirl is used here instead of Kasner's whirl because it is necessary to distinguish this from other types of whirl that appear below. This term was first used, for a similar reason, in J. M. Feld's Whirl-similitudes, euclidean kinematics, and non-euclidean geometry. (This Bulletin, abstract 46-5-270.) 
Let $\alpha=\alpha_{0} \mathfrak{e}_{0}+\alpha_{1} \mathfrak{e}_{1}+\alpha_{2} \mathfrak{e}_{2}+\alpha_{3} \mathfrak{e}_{3}$ and $N(\alpha)=1$; then the turn $T_{\alpha}$ is given by the equations:

$$
z^{*}=z, \quad \zeta^{*}=\alpha \zeta .
$$

The direct whirl $T_{\alpha} S_{a} T_{\beta}$ is given by the equations:

$$
z^{*}=\bar{\zeta} \bar{\alpha} a \alpha \zeta, \quad \zeta^{*}=\beta \alpha \zeta .
$$

If we let $\bar{\alpha} a \alpha=c$ and $\beta \alpha=\gamma$, the equations of a generic direct whirl $W_{c, \gamma}^{+}$ assume the form:

$$
z^{*}=z+\bar{\zeta} c \zeta, \quad \zeta^{*}=\gamma \zeta
$$

where $c$ is a vector and $\gamma$ is a unit quaternion.

We can now state the following theorems:

THEOREM 1. The slides constitute a continuous two-parameter abelian group such that $S_{a} \cdot S_{b}=S_{a+b}$.

THEOREM 2. The turns constitute a continuous three-parameter group such that $T_{\alpha} \cdot T_{\beta}=T_{\beta \alpha}$.

THEOREM 3. The direct whirls constitute a continuous six-parameter group. A necessary and sufficient condition that $W_{c, \gamma}^{+}=W_{a, \alpha}^{+} \cdot W_{b, \beta}^{+}$is that $c=a+\bar{\alpha} b \alpha$ and $\gamma=\beta \alpha$.

We shall let $\mathfrak{W}_{6}^{+}$represent the group of direct whirls. Noting that the direct whirls $W_{a, \alpha}^{+}$where $\alpha=e_{0}, a=k e_{3}$ ( $k$ real), form a one-parameter continuous group of contact transformations-namely, the group of dilatations $D_{k}$-we obtain the following theorem:

THEOREM 4. Direct whirls can be uniquely represented in the form $W_{c, \gamma}^{+}=D_{k} \cdot S_{b} \cdot T_{\alpha}$ where $c=k e_{3}+b, \gamma=\alpha$.

From equations (2.4) we obtain, by multiplying each member of the first equation on the left by the corresponding member of the second equation and on the right by the conjugate of the corresponding member of the second equation, $\zeta^{*} z^{*} \bar{\zeta}^{*}=\gamma(\zeta z \bar{\zeta}) \bar{\gamma}+\gamma c \bar{\gamma}$. Letting $Z^{*}=\zeta^{*} z^{*} \bar{\zeta}^{*}, Z=\zeta z \bar{\zeta}, \alpha=\bar{\gamma}, a=\gamma c \bar{\gamma}$, we have

$$
Z^{*}=\bar{\alpha} Z \alpha+a,
$$

which represents a euclidean point displacement $Z \rightarrow Z^{*}$ in $S_{3}$. Let this displacement be represented by $D_{a, \alpha}^{+}$. Then we obtain this theorem:

THEOREM 5. The group of direct whirls is simply isomorphic to the group of euclidean displacements in $S_{3}$ : the correspondence between members of these groups is given by $D_{a, \alpha}^{+} \leftrightarrow W_{b, \beta}^{+}$where $b=\alpha a \bar{\alpha}$ and $\beta=\bar{\alpha}$. 
3. The group of whirl-motions. Equation (2.5), which represents a point transformation, can be extended to represent a plane element transformation $(z, \zeta) \rightarrow\left(z^{*}, \zeta^{*}\right)$ as follows:

$$
z^{*}=\bar{\alpha} z \alpha+a, \quad \zeta^{*}=\zeta \alpha .
$$

Henceforth, $D_{a, \alpha}^{+}$shall designate the euclidean displacement of plane elements given by (3.1); $\mathfrak{D}_{6}^{+}$shall designate the six-parameter group of these displacements. The product of a displacement $D_{a, \alpha}^{+}$and a direct whirl $W_{b, \beta}^{+}$, in either order, shall be called a direct whirl-motion. The equations of the direct whirl-motion $D_{a, \alpha}^{+} \cdot W_{b, \beta}^{+}$are:

$$
z^{*}=\bar{\alpha}(z+\bar{\zeta} b \zeta) \alpha+a, \quad \zeta^{*}=\beta \zeta \alpha .
$$

Evidently $D_{a, \alpha}^{+} \cdot W_{b, \beta}^{+}=W_{b, \beta}^{+} \cdot D_{a, \alpha}^{+}$. The direct whirl-motions constitute a continuous twelve-parameter group, which shall be designated by $\left(\mathfrak{S}_{12}^{1}\right.$.

Let $l$ and $r$ be an arbitrary pair of vectors, and let the plane element $\left(\xi:\left(l+r, e_{0}\right)\right.$ be subjected to the $\infty^{3}$ possible rotations around the point whose position vector is equal to $l$; then the manifold of $\infty^{3}$ plane elements $(z, \zeta)$ into which $\xi$ is thereby transformed shall be called a (space) turbine. The equation of this turbine is

$$
z-l=\bar{\zeta} r \zeta \text {. }
$$

Since the vectors $l$ and $r$ completely characterize the turbine (3.3), we shall call $l$ and $r$ its left and right coordinates respectively, and let the symbol $[l, r]$ represent the turbine whose equation is (3.3). A brief computation will verify this theorem:

THEOREM 6. Turbines are transformed into turbines under $\$_{12}^{1}$. Under $\mathfrak{W}_{6}{ }^{+}$their left coordinates remain invariant; under $\mathfrak{D}_{6}^{+}$their right coordinates remain invariant.

Let us now consider a space analogue of the flat field, a concept that appears in Kasner's whirl-motion geometry in the plane. We shall call this analogue a dual element because it will serve as the dual of the plane element, just as the flat field serves as the dual of the lineal element. ${ }^{7}$ Dual elements shall be represented by two coordinates $s$ and $\sigma$ where the former is a vector and the latter is a unit quaternion; in order to distinguish dual elements from plane elements, the former shall be represented by the symbol $\{s, \sigma\}$. We define dual elements by imposing upon them the requirement that the turbine $[l, r]$ which, in plane element coordinates $z, \zeta$, has the equation (3.3) shall, in dual coordinates $s, \sigma$, have the equation

\footnotetext{
${ }^{7}$ Feld, loc. cit.
} 


$$
s-l=-\bar{\sigma} r \sigma .
$$

A turbine $[l, r]$ and a plane element $(z, \zeta)$ shall be said to be incident if their coordinates are connected by equation (3.3); similarly, $[l, r]$ and $\{s, \sigma\}$ shall be said to be incident if their coordinates are connected by equation $\left(3.3^{*}\right)$.

It is evident that direct whirls and displacements of plane elements induce contragredient transformations of the dual elements; for example, the dual element transformation contragredient to $W_{a, \alpha}^{+}$is

$$
s^{*}=s-\bar{\sigma} a \sigma, \quad \sigma^{*}=\alpha \sigma .
$$

This can be regarded, of course, as a representation of $W_{a, \alpha}^{+}$in dual element coordinates; likewise, displacements and whirl-motions can be expressed in dual element coordinates.

Let $\Im_{1}$ represent the involutory plane element transformation

$$
z^{*}=-z, . \quad \zeta^{*}=\zeta,
$$

which induces the dual element transformation

$$
s^{*}=-s, \quad \sigma^{*}=\sigma .
$$

The $\infty^{6}$ transformations $\Im_{1} \mathfrak{B}_{6}^{+}$or $\mathfrak{M}_{6}^{+} \Im_{1}$ shall be called opposite whirls; the family of opposite whirls shall be designated by $\mathfrak{B}_{\overline{6}}^{-}$. Similarly, the family $\Im_{1} \mathfrak{D}_{6}^{+} \equiv \mathfrak{D}_{6}^{+} \Im_{1}$, which embraces the $\infty^{6}$ euclidean symmetries in $S_{3}$, shall be designated by $\mathfrak{D}_{6}^{-}$; and the twelve-parameter family yielded by the products of $\Im_{1}$ and the direct whirl-motions shall be called the family of opposite whirl-motions, and shall be represented by $\mathfrak{S}_{12}^{2}$. Evidently, $\mathfrak{D}_{6}^{-} \mathfrak{2}_{6}{ }^{+} \equiv \mathfrak{D}_{6}^{+} \mathfrak{B}_{6} \equiv \mathfrak{S}_{12}^{2}$. Since the product of two opposite whirl-motions is a direct whirl-motion, the two families $\mathfrak{S}_{12}^{1}$ and $\mathfrak{S}_{12}^{2}$ form a mixed group.

Let $\Im_{2}$ represent the involutory correlation $(z, \zeta) \leftrightarrow\{s, \sigma\}$ given by the equations:

$$
z=s, \quad \zeta=\sigma .
$$

Let the family of transformations embraced by $\Im_{2} \mathfrak{S}_{12}^{1} \equiv\left(\mathfrak{S}_{12}^{1} \Im_{2}\right.$ be represented by $\mathfrak{S}_{12}^{3}$; likewise, let $\Im_{2} \mathfrak{S}_{12}^{2} \equiv \mathfrak{S}_{12}^{2} \Im_{2}$ be represented by $\mathfrak{S}_{12}^{4}$. The four families $\mathbb{S}_{12}^{i},(i=1,2,3,4)$, constitute a mixed group, which shall be designated by $\Gamma_{12}$ and called the group of proper whirl-motions.

Let $\Im_{3}$ represent the involutory plane element transformation $(z, \zeta) \rightarrow\left(z^{*}, \zeta^{*}\right)$ given by the equations:

$$
z^{*}=\zeta z \bar{\zeta}, \quad \zeta^{*}=\bar{\zeta} .
$$

This transformation induces the dual element transformation

$$
s^{*}=-\sigma s \bar{\sigma}, \quad \sigma^{*}=\bar{\sigma} .
$$


Let the products $\Im_{3} \mathfrak{S}_{12}^{i} \equiv \mathfrak{S}_{12}^{l} \Im_{3},(i=1,2,3,4)$, be designated by $\mathfrak{S}_{12}^{i}$. The four families $\mathfrak{S}_{12}^{i}$ embrace the improper whirl-motions. The proper and improper whirl-motions constitute a mixed twelve-parameter group composed of eight mutually exclusive families; this group, which shall be designated by $\bar{\Gamma}_{12}$, shall be called the complete group of whirl-motions. The following theorems are now evident.

THEOREM 7. Turbines are transformed into turbines under the complete group of whirl-motions.

THEOREM 8. The turbine transformation effected by $\Im_{3}$ is given by $l^{*}=r, r^{*}=l$.

4. The kinematic representation. Let the turbine $[l, r]$ be mapped on that ordered pair of points in $S_{3}$ whose position vectors are $l$ and $r$, in this order. By means of this mapping, which shall be known as the kinematic representation, ${ }^{8}$ a $(1,1)$ correspondence is set up between turbines $[l, r]$ and ordered point pairs $l, r$, the former of which is the left image point and the latter the right image point of $[l, r]$. If we regard, as we may, the $8 \infty^{12}$ whirl-motions in $\bar{\Gamma}_{12}$ as turbine transformations, $[l, r] \rightarrow\left[l^{*}, r^{*}\right]$, we find that the kinematic representation maps these transformations $(1,1)$ upon pairs of euclidean motions (displacements and symmetries) in $S_{3}$, which are either of the type $l \rightarrow l^{*}, r \rightarrow r^{*}$, or else of the type $l \rightarrow r^{*}, r \rightarrow l^{*}$. The pairs of motions of the former type correspond to the proper whirl-motions, and those of the latter type to the improper whirl-motions. Explicitly, the kinematic images of the families $\mathfrak{H}_{12}^{i}$ are as follows:

$$
\begin{array}{lll}
\mathfrak{H}_{12}^{1}: & l^{*}=\bar{\alpha} l \alpha+a, & r^{*}=\bar{\beta} r \beta+b, \\
\mathfrak{S}_{12}^{2}: & l^{*}=-\bar{\alpha} l \alpha+a, & r^{*}=-\bar{\beta} r \beta+b, \\
\mathfrak{S}_{12}^{3}: & l^{*}=\bar{\alpha} l \alpha+a, & r^{*}=-\bar{\beta} r \beta+b, \\
\mathfrak{S}_{12}^{4}: & l^{*}=-\bar{\alpha} l \alpha+a, & r^{*}=\bar{\beta} r \beta+b,
\end{array}
$$

$\alpha$ and $\beta$ are unit quaternions; $a$ and $b$ are vectors.

To obtain the kinematic image of the family $\mathfrak{S}_{12}^{i}$ we need but, in ac-

${ }^{8} \mathrm{~A}$ similar representation of whirl-motion geometry in the plane has been given by Feld, loc. cit. See also, in this connection, J. Grünwald's Ein Abbildungsprinzip, welches die ebene Geometrie und Kinematik mit der räumlichen Geometrie verknüpft, Sitzungsberichte der Akademie der Wissenschaften, Vienna, Mathematisch-Naturwissenschaftliche Klasse (II A), vol. 80 (1911), pp. 677-741; and two contributions by W. Blaschke, Euklidische Kinematik und nichteuklidische Geometrie, Zeitschrift für Mathematik und Physik, vol. 60 (1911), pp. 61-91, 203-204; and Ebene Kinematik, Berlin, 1938. 
cordance with Theorem 8, interchange $l$ and $r$ in the pair of equations given by (4.1) which corresponds to $\$_{12}^{i}$.

Inasmuch as the turbine $[l, r]$ has for its equation in plane element coordinates $z, \zeta: l=-\bar{\zeta} r \zeta+z$, and for its equation in dual element coordinates $s, \sigma: l=\bar{\sigma} r \sigma+s$, it is apparent that a necessary and sufficient condition that $[l, r]$ be incident to a given plane element $(z, \zeta)$ [dual element $\{s, \sigma\}]$ is that the left and right image points of $[l, r]$ in the kinematic representation be corresponding points in a euclidean symmetry [displacement]. Consequently, the kinematic representation establishes a $(1,1)$ correspondence between plane elements [dual elements] and euclidean symmetries [displacements] in $S_{3}$.

The kinematic mapping of plane elements and dual elements upon symmetries and displacements, respectively, furnishes us with the means of representing parametrically the composition of euclidean motions in $S_{3} .{ }^{9}$ An example will suffice to show how this can be done. Let the coordinates of the dual element $\{s, \sigma\}$ represent the displacement that corresponds to $\{s, \sigma\}$ in the kinematic representation; let the displacement corresponding to $\left\{s_{3}, \sigma_{3}\right\}$ be equivalent to the product of the displacements corresponding to $\left\{s_{1}, \sigma_{1}\right\}$ and $\left\{s_{2}, \sigma_{2}\right\}$, and in this order. With these conventions it follows that

$$
s_{3}=\bar{\sigma}_{2} s_{1} \sigma_{2}+s_{2}, \quad \sigma_{3}=\sigma_{1} \sigma_{2}
$$

in a similar manner we can represent other combinations of euclidean motions.

5. The group of whirl-similitudes. Let $\subseteq$ represent the pair of similitudes:

$$
l^{*}=k l, \quad r^{*}=k r
$$

where $k$ is a positive real number, and $l, r$ are the kinematic image points of $[l, r]$. Let $\mathfrak{S S}_{13}^{i} \equiv \mathfrak{S}\left(\mathfrak{S}_{12}^{i}\right.$ and $\mathfrak{S}_{13}^{i} \equiv \mathfrak{S} \mathfrak{S}_{12}^{i}$. Then the eight thirteen-parameter families comprising $\mathfrak{S}_{13}^{i}$ and $\mathfrak{S}_{13}^{i},(i=1,2,3,4)$, constitute a mixed group $\bar{\Gamma}_{13}$-the complete group of whirl-similitudes. This group is the space analogue of the group of whirl-similitudes in the plane. ${ }^{10}$ Evidently, turbines are transformed into turbines under $\bar{\Gamma}_{13}$.

Brooklyn College

${ }^{9}$ E. Study has given such a representation by means of Clifford biquaternions, op. cit.

${ }^{10}$ Feld, op. cit. 